TITLE:

\title{
Nucleon-Deuteron Breakup Differential Cross Sections Derived from the Quark-Model NN Interaction
}

\section{$\operatorname{AUTHOR}(\mathrm{S}):$}

Fujiwara, Yoshikazu; Fukukawa, Kenji

\section{CITATION:}

Fujiwara, Yoshikazu ... [et al]. Nucleon-Deuteron Breakup Differential Cross Sections Derived from the Quark-Model NN Interaction. Few-Body Systems 2013, 54(1-4): 489-493

ISSUE DATE:

2013-03

URL:

http://hdl.handle.net/2433/169912

\section{RIGHT:}

The final publication is available at www.springerlink.com; This is not the published version. Please cite only the published version.; この論文 は出版社版でありません。引用の際には出版社版をご確認ご利用くだ さい。 


\title{
Nucleon-Deuteron Breakup Differential Cross Sections Derived from the Quark-Model $N N$ Interaction
}

Received: date / Accepted: date

\begin{abstract}
The $n d$ and $p d$ breakup differential cross sections for $E_{N} \leq 65 \mathrm{MeV}$ are examined using the energy-independent quark-model nucleon-nucleon interaction fss2. The Coulomb effect is incorporated by the sharp cut-off Coulomb force, acting between quarks, without the phase-shift renormalization for the breakup amplitudes. Our model yields the results very similar to the meson-exchange potentials, including disagreement for some specific kinematical configurations. This includes the notorious space star anomaly of the $n d$ and $p d$ scattering at $E_{N}=13 \mathrm{MeV}$. The KVI data for the breakup differential cross sections of $E_{d}=130 \mathrm{MeV} d p$ scattering are reasonably reproduced by taking the Coulomb cut-off radius $\rho=16 \mathrm{fm}$.
\end{abstract}

Keywords Nucleon-deuteron scattering · Quark model nucleon-nucleon interaction

\section{Introduction}

In spite of the great success of rigorous three-body approaches [1] to the neutron-deuteron $(n d)$ and proton-deuteron $(p d)$ scattering, some three-nucleon $(3 N)$ observables are not well reproduced in $E_{N} \leq$ $65 \mathrm{MeV}$ region even with the recent accurate treatment of the Coulomb force [2, 3]. This is particularly true for nucleon-induced deuteron breakup processes. It is therefore worthwhile to reexamine the $N N$ interaction itself if the present-day realistic force is the most appropriate one to start with.

In previous studies [4, 5], we have applied the quark-model (QM) baryon-baryon interaction fss2 to problems of neutron-deuteron $(n d)$ elastic scattering. This interaction model fss2 [6] describes available $N N$ data in a comparable accuracy with the modern meson-exchange potentials. By eliminating the inherent energy dependence of the resonating-group kernel, fss 2 was found to yield a nearly correct triton binding energy, $S$-wave $n d$ scattering lengths and low-energy eigenphase shifts without introducing the $3 N$ force $[7,8]$. The so-called $A_{y}$ puzzle at low energies $E_{n} \leq 25 \mathrm{MeV}$ is somewhat improved in this model [5]. In this study, we examine $3 N$ breakup differential cross sections for various decaying kinematics in the energy range of $E_{n} \leq 65 \mathrm{MeV}$ [9]. Our main motivation is to determine if the quite different off-shell properties, originating from the strong nonlocality of the QM baryon-baryon interaction, affect the $3 N$ breakup differential cross sections. In contrast to the elastic scattering amplitude, the breakup amplitude covers a wide momentum region of the three-body phase space. It is found unfortunately that fss 2 gives predictions similar to the meson-exchange potentials and does not improve much the discrepancies between the theoretical and experimental results.

Y. Fujiwara and K. Fukukawa

Department of Physics, Kyoto University, Kyoto 606-8502, Japan

E-mail: yfujiwar@scphys.kyoto-u.ac.jp 


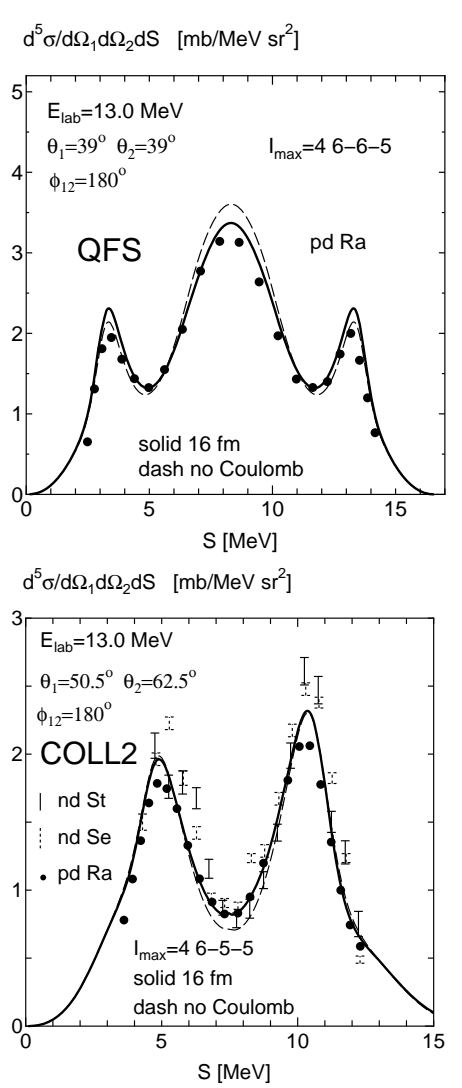

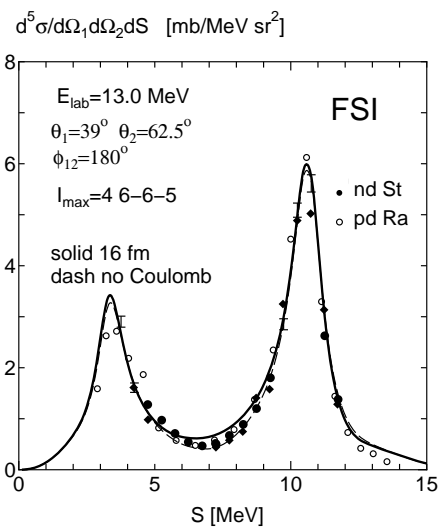

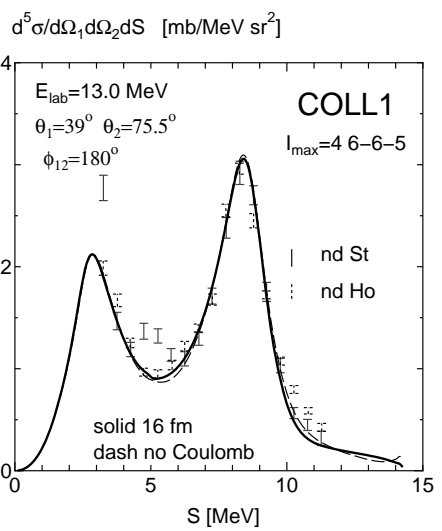

$$
\mathrm{d}^{5} \sigma / \mathrm{d} \Omega_{1} \mathrm{~d} \Omega_{2} \mathrm{dS} \quad\left[\mathrm{mb} / \mathrm{MeV} \mathrm{sr}{ }^{2}\right]
$$

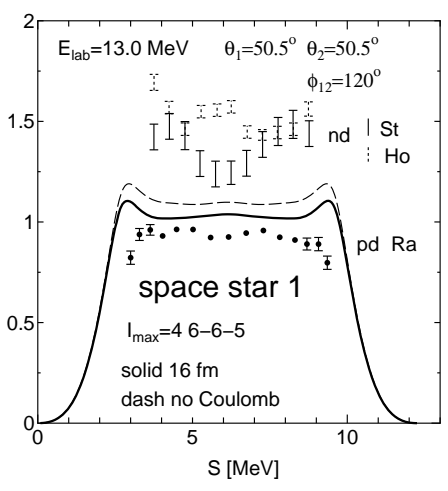

$\mathrm{d}^{5} \sigma / \mathrm{d} \Omega_{1} \mathrm{~d} \Omega_{2} \mathrm{dS} \quad\left[\mathrm{mb} / \mathrm{MeV} \mathrm{sr}{ }^{2}\right]$

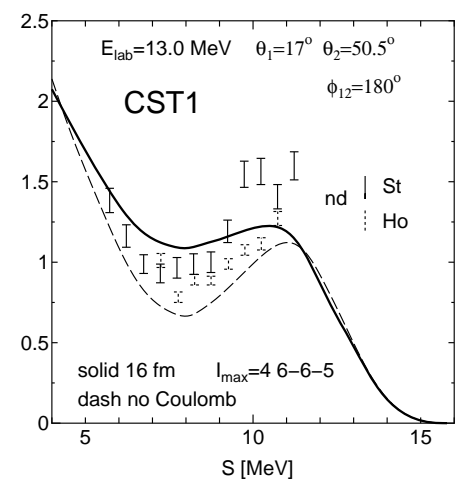

Fig. 1 Breakup differential cross sections for the reactions $d(p, 2 p) n$ (solid curve) and $d(n, 2 n) p$ (dashed curve) at $E_{N}=13 \mathrm{MeV}$, compared with the experimental data. The cut-off Coulomb radius $\rho=16 \mathrm{fm}$ is used.

\section{Coulomb treatment}

In the present calculation, the Coulomb force is treated as the three-body symmetric operator in the isospin formalism. The breakup amplitudes of the ppn system acquire an almost common phase factor, which does not contribute too much to the breakup differential cross sections. We therefore neglect this phase factor as a first step and calculate the breakup differential cross sections directly using the Coulomb modified breakup amplitudes. The Coulomb cut-off radius is chosen to be $\rho=16 \mathrm{fm}$ and $I_{\max }=4$ with $n_{1}-n_{2}-n_{3}=6-6-5$ is used in the notation of Ref. [4].

\section{Results}

Figure 1 shows the breakup differential cross sections for the reactions $d(p, 2 p) n$ (solid curve) and $d(n, 2 n) p$ (dashed curve) with the nucleon incident lab. energy $E_{N}=13 \mathrm{MeV}$. We find that the Coulomb effect is rather small in these examples and our results are very similar to the predictions by the meson-exchange potentials, given in Refs. [1, 2, 10]. A slight overestimation of the peak in quasi-free scattering (QFS) is reduced by the Coulomb effect and the agreement with the $p d$ experimental data [11] or more recent one [12] from Kyushu university group is improved. The $n p$ final state interaction (FSI) peaks are well reproduced. In the collinear (COLL1, COLL2) and coplanar cases, the nd data [13] agree well with theoretical predictions. The space star result is located just between the lower $p d$ data and the higher $n d$ data, which is the same feature as other predictions by the meson-exchange potentials. This disagreement of breakup differential cross sections at $E_{n}=13 \mathrm{MeV}$ was reported a long time ago, and is still an unsolved problem called space star anomaly [13].

We compare in Fig. 2 the breakup differential cross sections for the reaction $H(d, 2 p) n$ at $E_{d}=16$ $\mathrm{MeV}$ (solid curves) with the experimental data [14]. The Coulomb effect from the dashed curves (no 

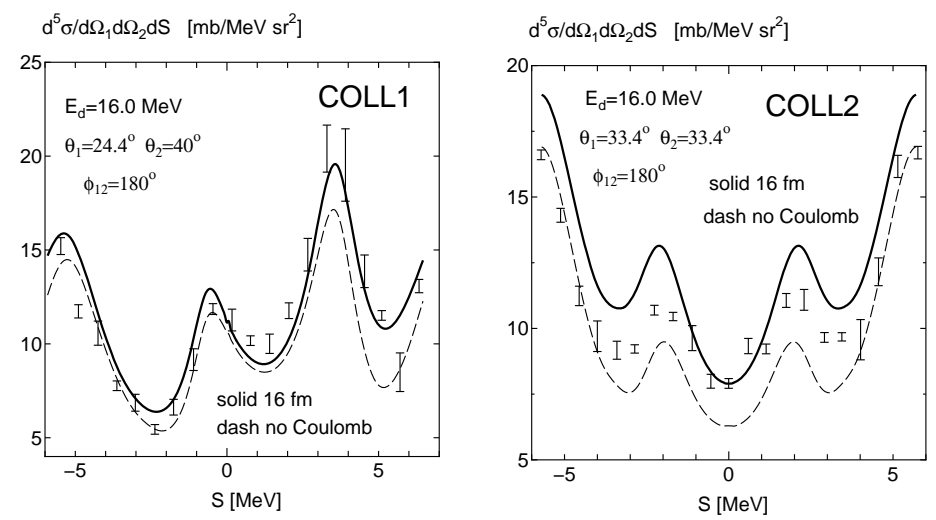

$\mathrm{d}^{5} \sigma / \mathrm{d} \Omega_{1} \mathrm{~d} \Omega_{2} \mathrm{dS} \quad\left[\mathrm{mb} / \mathrm{MeV} \mathrm{sr}{ }^{2}\right]$

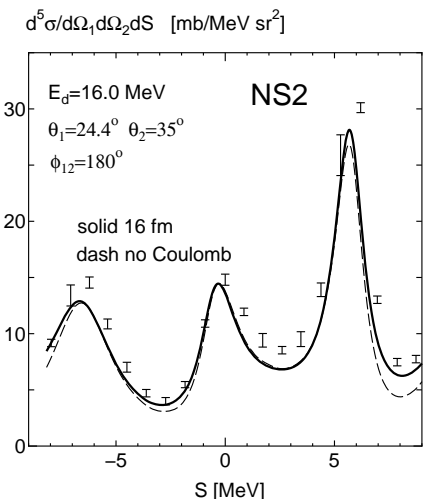

Fig. 2 Breakup differential cross sections for the reaction $H(d, 2 p) n$ with $E_{d}=16 \mathrm{MeV}$ (solid curve), compared with the experimental data [14]. The cut-off Coulomb radius $\rho=16 \mathrm{fm}$ is used.

Coulomb) to the solid curves (with Coulomb) improves the agreement with the experimental data in collinear (COLL1, COLL2) and the non-standard (NS1) configurations, although not perfect.

Very accurate KVI data for the $H(d, 2 p) n$ reaction with $E_{d}=130 \mathrm{MeV}[15]$ are compared with our predictions in Fig. 3. Here we find that the $p d$ calculations with the Coulomb effect (solid curves) generally improve the agreement with experiment, although the dip structure seen at the $p p$ final state interaction in Fig. 3(a) $\left(\theta_{1}=\theta_{2}=13^{\circ}\right.$ and $\left.\phi_{12}=20^{\circ}\right)$ is not sufficiently suppressed for $\rho=16 \mathrm{fm}$. A more accurate treatment of the Coulomb force, reproducing the correct low-energy behaviour of the half-off shell $p p t$-matrix, may be necessary for the improvement [15]. Too large cross section peaks in Figs. 3(a),(b) and the underestimation in Figs. 3(g) - (i) with the large azimuthal angles $\phi_{12} \sim 180^{\circ}$ may not be ascribed to the flaw of the Coulomb treatment.

\section{Summary}

We have applied our energy-independent quark-model nucleon-nucleon interaction fss 2 to the $n d$ and $p d$ breakup differential cross sections for $E_{N} \leq 65 \mathrm{MeV}$. The Coulomb effect is incorporated in the screened Coulomb approach without the phase-shift renormalization. This procedure seems to give reasonable results in the case of rather moderate Coulomb effects. An exception of this rule is the space star anomaly at $E_{N}=13 \mathrm{MeV}$, in which the effect of the Coulomb force is small and yet a big difference of the $n d$ and $p d$ data is empirically observed.

Acknowledgements This work was supported by the Grants-in-Aid for Scientific Research (C) (Grant No. 23540302) and for the Global COE Program "The Next Generation of Physics, Spun from Universality and Emergence" from the Ministry of the Education, Culture, Sports, Science and Technology (MEXT) of Japan. The numerical calculations were carried out on SR16000 at YITP in Kyoto University and on the highperformance computing system Intel Xeon X5680 at RCNP in Osaka University. 

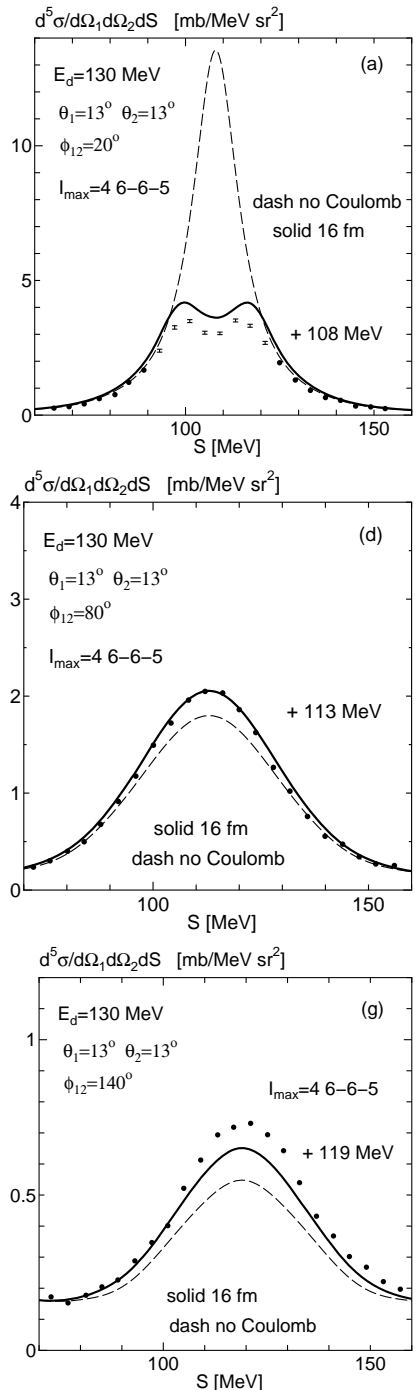
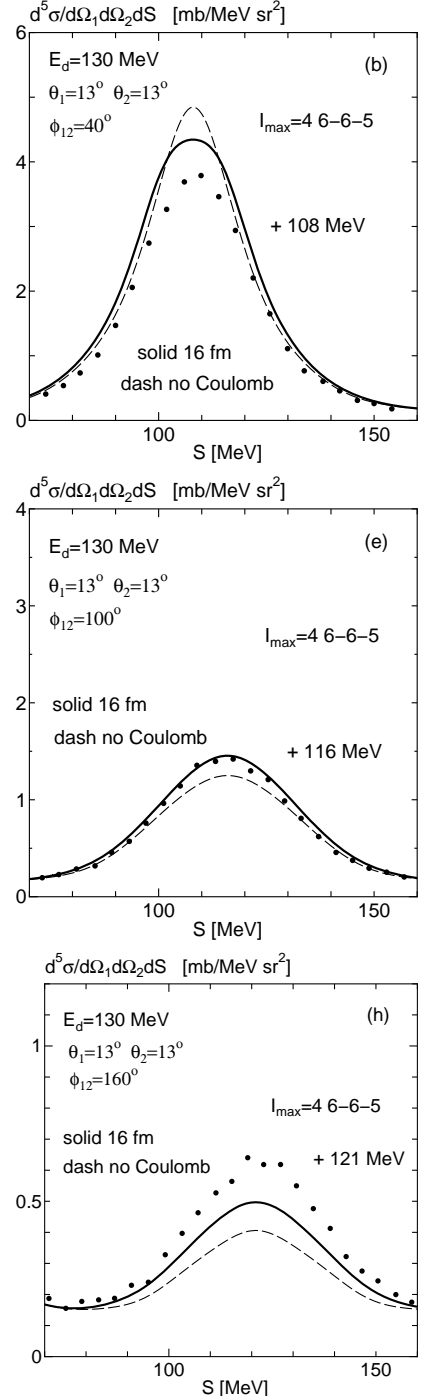
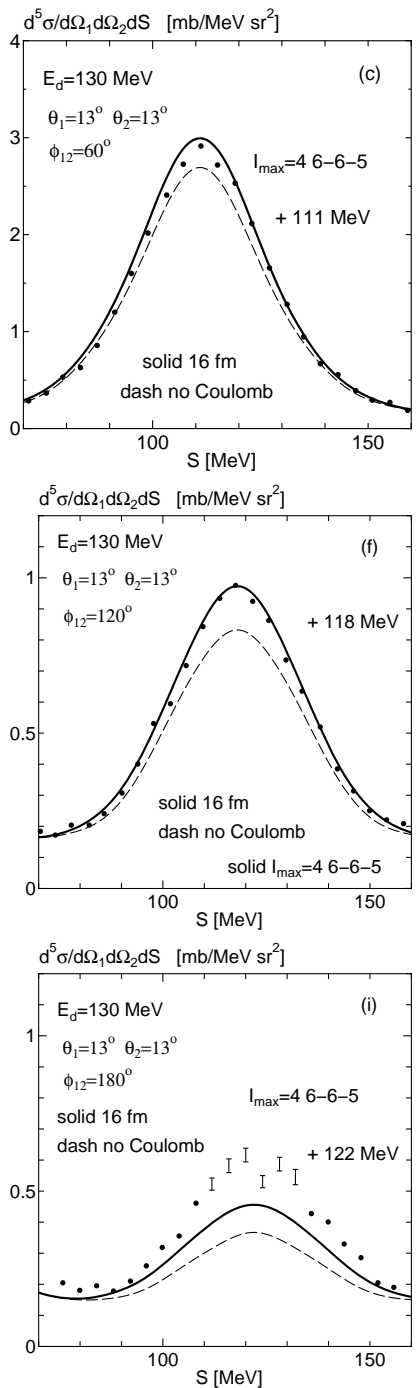

Fig. 3 Breakup differential cross sections for the reaction $H(d, 2 p) n$ with $E_{d}=130 \mathrm{MeV}$ (solid curves), compared with the experimental data [15]. Here $\theta_{1}=\theta_{2}=13^{\circ}$ and $\phi_{12}$ are changed from $20^{\circ}$ to $180^{\circ}$ with a step of $20^{\circ}$. The calculated curves are shifted by about $100 \mathrm{MeV}$ with respect to $S$ to fit the experimental origin. The cut-off Coulomb radius $\rho=16 \mathrm{fm}$ is used.

\section{References}

1. Glöckle, W., Witała, H., Hüber, D., Kamada, H., Golak, J.: The three nucleon continuum: achievements, challenges and applications. Phys. Rep. 274, 107 (1996)

2. Deltuva, A., Fonseca, A.C., Sauer, P.U.: Momentum-space treatment of the Coulomb interaction in threenucleon reactions with two protons Phys. Rev. C 71, 054005 (2005)

Deltuva, A., Fonseca, A.C., Sauer, P.U.: Momentum-space description of three-nucleon breakup reactions including the Coulomb interaction. Phys. Rev. C 72, 054004 (2005)

3. Ishikawa, S.: Coordinate space proton-deuteron scattering calculations including Coulomb force effects. Phys. Rev. C 80, 054002 (2009) and private communications

4. Fujiwara, Y., Fukukawa, K.: Quark-model baryon-baryon interaction applied to neutron-deuteron scattering. I - Noyes-Kowalski approach to the AGS equations - . Prog. Theor. Phys. 124, 433 (2010)

5. Fukukawa, K., Fujiwara, Y.: Quark-model baryon-baryon interaction applied to neutron-deuteron scattering. II - Polarization observables of the elastic scattering - . Prog. Theor. Phys. 125, 729 (2011)

6. Fujiwara, Y., Suzuki, Y., Nakamoto, C.: Baryon-baryon interactions in the $S U_{6}$ quark model and their applications to light nuclear systems. Prog. Part. Nucl. Phys. 58, 439 (2007)

7. Fujiwara, Y., Miyagawa, K., Kohno, M., Suzuki, Y., Nemura, H.: Triton binding energy calculated from the $S U_{6}$ quark-model nucleon-nucleon interaction. Phys. Rev. C 66, 021001(R) (2002) 
Fujiwara, Y., Miyagawa, K., Kohno, M., Suzuki, Y.: Faddeev calculation of the hypertriton using the $S U_{6}$ quark-model nucleon-nucleon and hyperon-nucleon interactions. Phys. Rev. C 70, 024001 (2004)

Fujiwara, Y., Suzuki, Y., Kohno, M., Miyagawa, K.: Addendum to triton and hypertriton binding energies calculated from $S U_{6}$ quark-model baryon-baryon interactions. Phys. Rev. C 77, 027001 (2008)

8. Fukukawa, K., Fujiwara, Y.: Effective-range expansion of the nd scattering studied by a quark-model nonlocal Gaussian potential. Prog. Theor. Phys. 125, 957 (2011)

9. Fujiwara, Y., Fukukawa, K.: Quark-model baryon-baryon interaction applied to neutron-euteron scattering. III - Breakup differential cross sections - . Prog. Theor. Phys. 125, 979 (2011)

10. Kuroś-Żołnierczuk, J., et al.: Three-nucleon force effects in nucleon induced deuteron breakup. I. Predictions of current models. Phys. Rev. C 66, 024003 (2002)

Kuroś-Żołnierczuk, J., et al.: Three-nucleon force effects in nucleon induced deuteron breakup. II. Comparison to data. Phys. Rev. C 66, 024004 (2002)

11. Rauprich, G., et al.: Study of the kinematically complete breakup reaction ${ }^{2} \mathrm{H}(\vec{p}, p p) n$ at $E_{p}=130 \mathrm{MeV}$ with polarized protons. Nucl. Phys. A535, 313 (1991)

12. Kimura, S., et al.: Contribution to this conference

13. Setze, H.R., et al.: Verification of the space-star anomaly in $n d$ breakup. Phys. Rev. Lett. B388, 229 (1996)

14. Correll, F.D., et al.: Study of the reaction $\mathrm{H}(\vec{d}, 2 p) n$ at $16 \mathrm{MeV}$ around collinear configurations. Nucl. Phys. A475, 407 (1987)

15. Kistryn, St., et al.: Systematic study of three-nucleon force effects in the cross section of the deuteronproton breakup at $130 \mathrm{MeV}$. Phys. Rev. C 72, 044006 (2005)

Kistryn, St., et al.: Evidence of the Coulomb-force effects in the cross-sections of the deuteron-proton breakup at $130 \mathrm{MeV}$. Phys. Lett. B641, 23 (2006) 